\title{
Comparing Zinc Oxide- and Zinc Silicate-Related Metal-Organic Networks via Connection-Based Zagreb Indices
}

\author{
Muhammad Tanveer Hussain, ${ }^{1}$ Muhammad Javaid (iD, ${ }^{1}$ Usman Ali, ${ }^{1}$ Ali Raza, \\ and Md Nur Alam $\mathbb{D}^{2}$ \\ ${ }^{1}$ Department of Mathematics, School of Science, University of Management and Technology, Lahore 54770, Pakistan \\ ${ }^{2}$ Department of Mathematics, Pabna University of Science and Technology, Pabna 6600, Bangladesh \\ Correspondence should be addressed to Md Nur Alam; nuralam23@pust.ac.bd
}

Received 13 August 2021; Revised 29 September 2021; Accepted 4 October 2021; Published 18 October 2021

Academic Editor: Ajaya Kumar Singh

Copyright (c) 2021 Muhammad Tanveer Hussain et al. This is an open access article distributed under the Creative Commons Attribution License, which permits unrestricted use, distribution, and reproduction in any medium, provided the original work is properly cited.

\begin{abstract}
Metal-organic networks (MONs) are among the unique complex and porous chemical compounds. So, these chemical compounds consist of metal ions (vertices) and organic ligands (edges between vertices). These networks represent large pore volume, extreme surface area, morphology, excellent chemical stability, highly porous and crystalline materials, and octahedral clusters. MONs are mostly used in assessment of chemicals, gas and energy storage devices, sensing, separation and purification of different gases, heterogeneous catalysis, environmental hazard, toxicology, adsorption analysis, biomedical applications, and biocompatibility. Recently, drug delivery, cancer imaging, and biosensing have been investigated by biomedical applications of zincrelated MONs. The versatile applications of these MONs make them helpful tools in many fields of science in recent decade. In this paper, we discuss the two different zinc oxide and zinc silicate related MONs according to the number of increasing layers of metal and organic ligands together. We also compute the connection-based Zagreb indices such as first Zagreb connection index (ZCI), second ZCI, modified first ZCI, modified second ZCI, modified third ZCI, and modified fourth ZCI. Moreover, a comparison is also included between the zinc-related MONs by using numerical values of connection-based Zagreb indices. Finally, we conclude that zinc silicate-related MON is better than zinc oxide-related MON for all values of $n$.
\end{abstract}

\section{Introduction}

There are so many chemical compounds in the field of chemistry. One of the most popular recent chemical compounds is metal-organic network (MON) which consists of metal ions and organic ligands. A new MON with zinc as the metal ion and benzene-1,3-dicarboxylic acid as the organic ligand (linker) has been synthesized with the help of hydrothermal method. This MON is also used as a selective nanoadsorbent for the preconcentration and extraction of trace amounts of cadmium with the help of solid-phase extraction method. A category of crystalline and porous materials is porous coordination polymers which are newly known as MONs [1]. One of the most important aspects which can be considered in the matter of MONs in bioapplications is their surface modification and control of their particle size distribution [2]. Zn-related MONs as chemical sensors could be converted into devices for luminescent characteristics [3]. The electron-rich T-conjugated fluorescent ligands are friendly to make $\mathrm{Zn}$ MONs with nucleophilic properties in efficient luminescent sensors [4].

The low toxicity of zinc ions as the desirable character is considered to introduce $\mathrm{Zn}$-related MONs into bioapplication domains, especially drug carries. The antibacterial activity has been validated by combining different antibiotic drugs and metals [5]. In reality, $\mathrm{Zn}^{2+}$ is an endogenous low-toxic transition metal cation which is widely used in dermatology as a cicatrizing agent and skin moisturizer with astringent, antidandruff, antibacterial, and anti-inflammatory agents [6]. In nonlinear optically active MONs, $\mathrm{Zn}^{2+}$ is commonly used as a connecting point 
to prevail undesired $\mathrm{d}-\mathrm{d}$ transitions in the visible region. Moreover, the toxicology, biomedical applications, and their biocompatibility are recently reported production procedures of zinc-related MONs. For more details, refer to [7].

Eddaoudi et al. [8] synchronized the isoreticular series (IRMOF-1 to IRMOF-16) of 16 highly crystalline materials. The free and fixed diameter of pores from IRMOF-1 to IRMOF-16 varies in the range of 3.8-19.1 and $12.8-28.8$, respectively. The design of an IRMOF-10 series based on MON-5 was initiated by determining the reaction conditions necessary to produce the octahedral cluster with a ditopic linear carboxylate. Therefore, many IRMOF structures can be developed by using zinc oxide octahedral clusters $\left(\mathrm{Zn}_{4} \mathrm{O}(\mathrm{CO})_{2}\right)$ as the metal corners linked via diverse organic dicarboxylate linkers and different three-dimensional cubic networks are formed. For more information, see [9]. All the IRMOFs have the expected topology of $\mathrm{CaB}_{6}(13)$ and happened through the prototype IRMOF-1 in which an oxide-centered $\mathrm{Zn}_{4} \mathrm{O}$ tetrahedron is edge bridged. Some IRMOFs such as IRMOF-8, IRMOF-10, IRMOF-12, and IRMOF-16 have been seen in noncrystalline porous systems for $\mathrm{SiO}_{2}$ xerogels and aerogels (16). For further investigation, see [7, 10-12].

MONs also predict the physicochemical properties such as grafting active groups [13], impregnating suitable active material [14], ion exchange [15], preparing composites with different substances [11], changing organic ligands and postsynthetic ligands [16], and biosensors enhancing sensitivity, response time, and selectivity [17]. Yap et al. [18] and Lin et al. [19] presented the recent progress in precursors on the preparation of several nanostructures and MON-related applications such as sensing, photocatalysis, electrocatalysis, supercapacitors, catalyst for production of fine chemicals, and lithium ion batteries. Graph theory provides useful tools in the field of modern chemistry which represent the chemical and physical properties of chemical compounds such as heat of formation, heat of evaporation, flash point, melting point, boiling point, temperature, pressure, density, retention in chromatography, and tension and partition coefficient [20-22]. First, distance-based topological index (TI) was discovered by Wiener to study the different properties of chemical compounds (boiling point of paraffin) in 1947 [23]. The very well-reputed first-degreebased TI was discovered by Gutman and Trinajstić to check the chemical physibility on the total $\pi$-electron energy of the chemical compounds (alternant hydrocarbons) in 1972 [24].

Recently, Zhao et al. [25] introduced two connection number (number of vertices at distance two) based TIs to compute the general results for modified Zagreb connection indices of subdivision and semitotal point operations on graphs. Nowadays, these degree and connection numberbased TIs are abundantly used in the topological properties of four-layered neural networks and MONs [26, 27]. Ali et al. [28] computed connection-based indices and coindices for the product of molecular networks. Gutman and Furtula discussed various topological properties of different molecular structures; see [29-31]. Ali and Trinajstic [32] and Javaid et al. [33] computed different connection-based TIs of graphs under different operations. Moreover, a variety of networks has been defined with the help of connection number-based TIs [34-37].

In this paper, we compute the connection-based Zagreb indices of two different zinc-related MONs such as zinc oxide (ZNOX $(n)=$ IRMOF-10) and zinc silicate (ZNCL $(n)=$ IRMOF-14) networks with respect to the increasing layers, $n \geq 3$, taking both metal nodes and linkers together. The rest of the paper is organized as follows. Section 2 provides the preliminaries, definitions, and some important results which can be used in the main results. Sections 3 and 4 provides the main results for zinc oxide and zinc silicate networks, and Section 5 provides comparisons and conclusions.

\section{Preliminaries}

The vertex and edge sets are $V(G)$ and $E(G)$ for a simple and connected network $G .|V(G)|$ and $|E(G)|$ are the cardinalities of vertex set and edge set which are equal to $u$ and $e$, respectively. In a connected network, there is a path between two vertices. The distance between two vertices $p$ and $q$ is the shortest path between them. It is denoted by $d_{G}(p, q)$. In general [37], $N_{G}(q / m)=\{p \in V(G) ; d(p, q)=m\}$ is the open $m$-neighborhood set of $q$, where $m$ represents a positive integer and $\left|N_{G}(q / m)\right|=d_{G}(q / m)$ is called $m$-distance degree of a vertex $q$. In particular,

(i) If $m=1, d_{G}(q / 1)=\left|N_{G}(q / 1)\right|=d_{G}(q)=$ degree of $q$ (number of vertices at distance one from $q$ )

(ii) If $m=2, d_{G}(q / 2)=\left|N_{G}(q / 2)\right|=\tau_{G}(q)=$ connection number of $q$ (number of vertices at distance two from q)

For more terminologies and notations, see [36] and references therein.

Definition 1. For a (molecular) network $G$, the first Zagreb index $\left(M_{1}(G)\right)$, second Zagreb index $\left(M_{2}(G)\right)$, and third Zagreb index $\left(M_{3}(G)\right)$ are defined as follows:

(a) $M_{1}(G)=\sum_{q \in V(G)}\left[d_{G}(q)\right]^{2}=\sum_{p q \in E(G)}\left[d_{G}(p)+d_{G}\right.$ $(q)]$

(b) $M_{2}(G)=\sum_{p q \in E(G)}\left[d_{G}(p) \times d_{G}(q)\right]$

(c) $M_{3}(G)=\sum_{q \in V(G)}\left[d_{G}(q)\right]^{3}=\sum_{p q \in E(G)}\left[d_{G}^{2}(p)+d_{G}^{2}\right.$ (q)]

These degree-based TIs are defined by Gutman and Trinajstic [24]. These are abundantly used to predict better findings in molecular networks such as ZE isomerism, absolute value of correlation coefficient, entropy, acentric factor, and heat capacity.

Definition 2. For a (molecular) network $G$, the first ZCI $\left(Z C_{1}(G)\right)$, second ZCI $\left(Z C_{2}(G)\right)$, and modified first ZCI $\left(Z C_{1}^{*}(G)\right)$ are defined as follows: 
(a) $Z C_{1}(G)=\sum_{q \in V(G)}\left[\tau_{G}(q)\right]^{2}$

(b) $Z C_{2}(G)=\sum_{p q \in E(G)}\left[\tau_{G}(p) \times \tau_{G}(q)\right]$

(c) $Z C_{1}^{*}(G)=\sum_{(p q \in E(G))}=\left[\tau_{G}(p)+\tau_{G}(q)\right]$

These connection-based TIs are defined by Ali and Trinajstic [32] (2018). They also reported that the modified first Zagreb connection index has better correlation coefficient value for the thirteen physicochemical properties of octane isomers than classical Zagreb indices.

Definition 3. For a (molecular) network $G$, the modified second Zagreb connection index $\left(Z C_{2}^{*}(G)\right)$ and modified third Zagreb connection index $\left(Z C_{3}^{*}(G)\right)$ are defined as follows:
(a) $Z C_{2}^{*}(G)=\sum_{p q \in E(G)}\left[d_{G}(p) \tau_{G}(q)+d_{G}(q) \tau_{G}(p)\right]$
(b) $Z C_{3}^{*}(G)=\sum_{p q \in E(G)}\left[d_{G}(p) \tau_{G}(p)+d_{G}(q) \tau_{G}(q)\right]$

Definition 4. For a (molecular) network $G$, the modified fourth Zagreb connection index $\left(Z C_{4}^{*}(G)\right)$ is defined as follows:

$$
Z C_{4}^{*}(G)=\sum_{p q \in E(G)}\left[d_{G}(p) \tau_{G}(p) \times d_{G}(q) \tau_{G}(q)\right] .
$$

These connection-based TIs are defined by Javaid et al. [35] to compute the exact solutions of several wheel-related graphs.

Definition 5. Zinc oxide network (ZNOX $(n)$ ): a chemical compound zinc oxide $(\mathrm{ZnO})$ is insoluble in water which is an inorganic compound of white powder shape with $5.61 \mathrm{~g} / \mathrm{cm}^{3}$ density. The zinc oxide is heated with carbon (coke) that reduces to the metal vapor to condense the liquid from which the solid metal freezes.

$$
\mathrm{ZnO}_{(s)}+\mathrm{C}_{(s)} \longrightarrow \mathrm{Zn}_{(g)}+\mathrm{CO}_{(g)}
$$

Zinc is a reactive metal to produce zinc ion $\left(\mathrm{Zn}^{2+}\right)$ and hydrogen gas. It also reduces those metal ions whose reduction potentials are higher than $\mathrm{Zn}^{2+}$. Zinc oxide is mostly used in making rubber, enamels, glazes, pigment in white paint, photoconductive surfaces, and protective coating for other metals. Zinc oxide-related $\mathrm{MON}$ is $\mathrm{Zn}_{4} \mathrm{O}(\mathrm{BPDC})_{3}$, which is also known as IRMOF-10. IRMOF-9 is a catenated version of IRMOF-10. IRMOF-10 is three-dimensional cubic structures with a pore size diameter of 16.7/20.2 $A^{0}$. In Figure 1, the zinc oxide-related MON of dimension 3 is presented. In general, the vertices and edges in ZNOX $(n)$ of dimension $n$ are $70 n+46$ and $85 n+55$, respectively. For more understanding, see Figure 1.

Definition 6. Zinc silicate network (ZNSL $(n)$ ): silicate $\left(\mathrm{SiO}_{4}\right)$ is the most charming class of minerals. Silicate is the

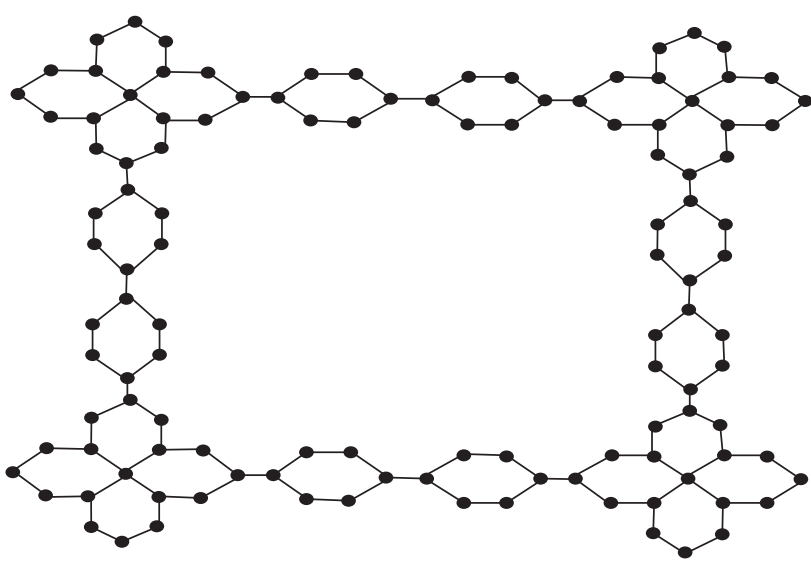

FIgURE 1: Zinc oxide network $(\operatorname{ZNOX}(n) \cong H)$ for $n=3$.

chemical mixture of metal oxide or metal carbonate with sand. The basic unit of silicate is tetrahedron. So, all silicates gain $\mathrm{SiO}_{4}$ tetrahedron. In chemistry, oxygen ions and silicon ions are represented by the corner vertices and centre vertices of $\mathrm{SiO}_{4}$, respectively. In graph theory, we represent corner vertices and centre vertices of $\mathrm{SiO}_{4}$ with oxygen nodes and silicon nodes. If we require a variety of silicate networks, it is easy to change the arrangement of the tetrahedron silicate. Zinc silicate-related $\mathrm{MON}$ is $\mathrm{Zn}_{4} \mathrm{O}$ (PDC) $)_{3}$, which is also known as IRMOF-14. IRMOF14 is three-dimensional cubic structures with a pore size diameter of 14.7/20.1 $A^{0}$. In Figure 2, the zinc silicate-related MON of dimension 3 is presented. In general, the vertices and edges in ZNSL $(n)$ of dimension $n$ are $82 n+50$ and $103 n+61$, respectively. For more understanding, see Figure 2.

Now, we present some important results which are used in the main results.

Lemma 1. Let $G$ be a connected network with $u$ vertices and e edges. Then, $\tau_{G}(p)+d_{G}(p) \leq \sum_{q \in N_{G}(p)} d_{G}(q)$, where equality holds if and only if $G$ is a $\left\{C_{3}, C_{4}\right\}$-free network.

Lemma 2 (see [35]). Let $G$ be a connected and $\left\{C_{3}, C_{4}\right\}$-free network with $u$ vertices and e edges. Then,

(i) $\sum_{q \in V(G)} d_{G}(q)=2 e$

(ii) $\sum_{q \in V(G)} \tau_{G}(q)=M_{1}(G)-2 e$

Lemma 3 (see [25]). Let $G$ be a connected and $\left\{C_{3}, C_{4}\right\}$-free network with $u$ vertices and e edges. Also, $G \cong P_{u}$. Then

(i) $Z C_{2}^{*}(G)=8 u-22$ if $u \geq 4$

(ii) $Z C_{3}^{*}(G)=8 u-22$ if $u \geq 3$ 


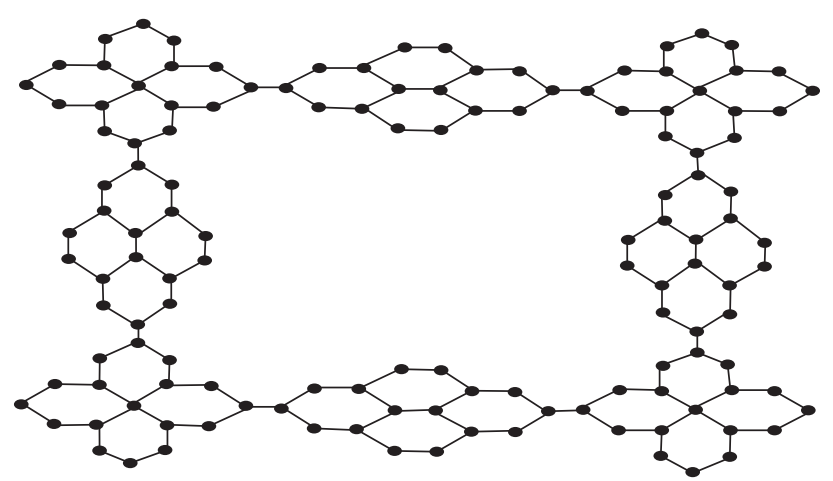

Figure 2: Zinc silicate network $(\operatorname{ZNSL}(n) \cong K)$ for $n=3$.

\section{Main Results Based on Zinc Oxide Network (ZNOX $(n))$}

In this section, we compute the main results for first Zagreb connection index (ZCI), second ZCI, modified first ZCI, modified second ZCI, modified third ZCI, and modified fourth ZCI of zinc oxide-related $\operatorname{MON}(\mathrm{ZNOX}(n))$. Let $H \cong$ $\operatorname{ZNOX}(n)$ be the zinc oxide network of dimension $n$ in the plane, see Figure 1. The partitions of $H$ with respect to the vertex set and edge set are $V(H)$ and $E(H)$. We can easily see each vertex of degrees 2, 3, and 4 . We have $V_{1}=\left\{v \in V(H) \mid d_{v}=2\right\}, \quad V_{2}=\left\{v \in V(H) \mid d_{v}=3\right\}, \quad$ and $V_{3}=\left\{v \in V(H) \mid d_{v}=4\right\}$, where $\left|V_{1}\right|=42 n+30, \quad\left|V_{2}\right|=$ $26 n+14$, and $\left|V_{3}\right|=2 n+2$. So, $|V(H)|=v=\left|V_{1}\right|+\left|V_{2}\right|+\mid$ $V_{3} \mid=70 n+46$. Now, the partitions of vertices according to connection number are $V_{1}=\left\{v \in V(H) \mid, \tau_{v}=2\right\}, V_{2}=\{v \in V$ $\left.(H) \mid \tau_{v}=3\right\}, \quad V_{3}=\left\{v \in V(H) \mid \tau_{v}=4\right\}, V_{4}=\left\{v \in V(H) \mid \tau_{v}=5\right\}$, and $V_{5}=\left\{v \in V(H) \mid \tau_{v}=8\right\}$, where $\left|V_{1}\right|=2 n+6, \quad\left|V_{2}\right|=$ $28 n+20,\left|V_{3}\right|=30 n+10,\left|V_{4}\right|=8 n+8$, and $\left|V_{5}\right|=2 n+2$. So, $\mid$ $V(H)|=v=| V_{1}|+| V_{2}|+| V_{3}|+| V_{4}|+| V_{5} \mid=70 n+46$. These vertex partitions are shown in Tables 1 and 2 .

There are four types of partitions of edge sets of $H$ according to the degree as $|E \quad(H)|=\left|E_{2,2}^{d}\right|+\left|E_{2,3}^{d}\right|+$ $\left|E_{3,3}^{d}\right|+\left|E_{3,4}^{d}\right|=85 n+55$, and there are seven types of partitions of edge sets of $H$ according to the connection number of vertices as $|E \quad(H)|=\left|E_{2,3}^{c}\right|+\left|E_{3,3}^{c}\right|+\left|E_{3,4}^{c}\right|+$ $\left|E_{3,5}^{c}\right|+\left|E_{4,4}^{c}\right|+\left|E_{4,5}^{c}\right|+\left|E_{5,8}^{c}\right|=85 n+55$. These edge partitions are shown in Tables 3 and 4 .
TABLe 1: Partitions of $H$ 's vertices according to the degree.

\begin{tabular}{lccc}
\hline$d_{v}$ & 2 & 3 & 4 \\
\hline$\left|d_{v}\right|$ & $42 n+30$ & $26 n+14$ & $2 n+2$ \\
\hline
\end{tabular}

TABle 2: Partitions of $H$ 's vertices according to the connection number.

\begin{tabular}{cccccc}
\hline$\tau_{v}$ & 2 & 3 & 4 & 5 & 8 \\
\hline$\left|\tau_{v}\right|$ & $2 n+6$ & $28 n+20$ & $30 n+10$ & $8 n+8$ & $2 n+2$ \\
\hline
\end{tabular}

Table 3: Partitions of $H$ 's edges according to the degree.

\begin{tabular}{lcccc}
\hline$E_{d(p), d(q)}^{d}$ & $E_{2,2}^{d}$ & $E_{2,3}^{d}$ & $E_{3,3}^{d}$ & $E_{3,4}^{d}$ \\
\hline$\left|E_{d(p), d(q)}^{d}\right|$ & $6 n+16$ & $52 n+28$ & $9 n+3$ & $8 n+8$ \\
\hline
\end{tabular}

Theorem 1. Let $H \cong Z N O X(n)$ be a zinc oxide network of dimensions $n \geq 3$. Then, the first Zagreb connection index is

$$
Z C_{1}(H)=1068 n+692 .
$$

Proof. By definition,

$$
\begin{aligned}
Z C_{1}(G) & =\sum_{q \in V(G)}\left[\tau_{G}(q)\right]^{2} \\
& =(2 n+6)(2)^{2}+(28 n+20)(3)^{2}+(30 n+10)(4)^{2}+(8 n+8)(5)^{2}+(2 n+2)(4)^{2} \\
& =8 n+24+252 n+180+480 n+160+200 n+200+128 n+128 \\
& =1068 n+692 .
\end{aligned}
$$

Theorem 2. Let $H \cong Z N O X(n)$ be a zinc oxide network of dimensions $n \geq 3$. Then, the second Zagreb connection index is

$$
Z C_{2}(H)=1376 n+896 .
$$


TABle 4: Partition of $H$ 's edges according to the connection number.

\begin{tabular}{lccccccc}
\hline$E_{\tau(p), \tau(q)}^{c}$ & $E_{2,3}^{c}$ & $E_{3,3}^{c}$ & $E_{3,4}^{c}$ & $E_{3,5}^{c}$ & $E_{4,4}^{c}$ & $E_{4,5}^{c}$ & $E_{5,8}^{c}$ \\
\hline$\left|E_{\tau(p), \tau(q)}^{c}\right|$ & $4 n+12$ & $4 n+12$ & $24 n+12$ & $4 n+12$ & $21 n+7$ & $12 n+4$ & $8 n+8$ \\
\hline
\end{tabular}

Proof. By definition,

$$
\begin{aligned}
Z C_{2}(G)= & \sum_{p q \in E(G)}\left[\tau_{G}(p) \times \tau_{G}(q)\right] \\
= & \sum_{p q \in E_{2,3}^{c}}\left[\tau_{H}(p) \times \tau_{H}(q)\right]+\sum_{p q \in E_{3,3}^{c}}\left[\tau_{H}(p) \times \tau_{H}(q)\right]+\sum_{p q \in E_{3,5}^{c}}\left[\tau_{H}(p) \times \tau_{H}(q)\right] \\
& +\sum_{p q \in E_{4,5}^{c}}\left[\tau_{H}(p) \times \tau_{H}(q)\right]+\sum_{p q \in E_{4,4}^{c}}\left[\tau_{H}(p) \times \tau_{H}(q)\right]+\sum_{p q \in E_{3,4}^{c}}\left[\tau_{H}(p) \times \tau_{H}(q)\right] \\
& +\sum_{p q \in E_{4,4}^{c}}\left[\tau_{H}(p) \times \tau_{H}(q)\right]+\sum_{p q \in E_{5,8}^{c}}\left[\tau_{H}(p) \times \tau_{H}(q)\right] \\
= & \left|E_{(2,3)(H)}\right|(2 \times 3)+\left|E_{(3,3)(H)}\right|(3 \times 3)+\left|E_{(3,5)(H)}\right|(3 \times 5)+\left|E_{(4,5)(H)}\right|(4 \times 5) \\
& +\left|E_{(4,4)(H)}\right|(4 \times 4)+\left|E_{(3,4)(H)}\right|(3 \times 4)+\left|E_{(4,4)(H)}\right|(4 \times 4)+\left|E_{(5,8)(H)}\right|(5 \times 8) \\
= & (4 n+12)(2 \times 3)+(12 n+4)(3 \times 3)+(4 n+12)(3 \times 5)+(12 n+4)(4 \times 5) \\
& +(12 n+4)(4 \times 4)+(24 n+8)(3 \times 4)+(9 n+3)(4 \times 4)+(8 n+8)(5 \times 8) \\
= & 24 n+72+108 n+36+60 n+180+240 n+80+192 n+64 \\
& +288 n+96+144 n+48+320 n+320 \\
= & 1376 n+896 .
\end{aligned}
$$

Theorem 3. Let $H \cong Z N O X(n)$ be a zinc oxide network of dimensions $n \geq 3$. Then, the modified first Zagreb connection index is

$$
Z C_{1}^{*}(H)=672 n+432 .
$$

Proof. By definition,

$$
\begin{aligned}
Z C_{1}^{*}(G)= & \sum_{p q \in E(G)}\left[\tau_{G}(p)+\tau_{G}(q)\right] \\
= & \sum_{p q \in E_{2,3}^{c}}\left[\tau_{H}(p)+\tau_{H}(q)\right]+\sum_{p q \in E_{3,3}^{c}}\left[\tau_{H}(p) \times \tau_{H}(q)\right]+\sum_{p q \in E_{3,5}^{c}}\left[\tau_{H}(p)+\tau_{H}(q)\right] \\
& +\sum_{p q \in E_{4,5}^{c}}\left[\tau_{H}(p) \times \tau_{H}(q)\right]+\sum_{p q \in E_{4,4}^{c}}\left[\tau_{H}(p) \times \tau_{H}(q)\right]+\sum_{p q \in E_{3,4}^{c}}\left[\tau_{H}(p) \times \tau_{H}(q)\right] \\
& +\sum_{p q \in E_{4,4}^{c}}\left[\tau_{H}(p) \times \tau_{H}(q)\right]+\sum_{p q \in E_{5,8}^{c}}\left[\tau_{H}(p) \times \tau_{H}(q)\right] \\
= & \left|E_{(2,3)(H)}\right|(2+3)+\left|E_{(3,3)(H)}\right|(3+3)+\left|E_{(3,5)(H)}\right|(3+5)+\left|E_{(4,5)(H)}\right|(4+5) \\
& +\left|E_{(4,4)(H)}\right|(4+4)+\left|E_{(3,4)(H)}\right|(3+4)+\left|E_{(4,4)(H)}\right|(4+4)+\left|E_{(5,8)(H)}\right|(5+8) \\
= & (4 n+12)(5)+(12 n+4)(6)+(4 n+12)(8)+(12 n+4)(9)+(12 n+4)(8) \\
& +(24 n+8)(7)+(9 n+3)(8)+(8 n+8)(13) \\
= & 20 n+60+72 n+24+32 n+96+108 n+36+96 n+32+168 n+56 \\
& +72 n+24+104 n+104 \\
= & 672 n+432 .
\end{aligned}
$$


Theorem 4. Let $H \cong Z N O X(n)$ be a zinc oxide network of dimensions $n \geq 3$. Then, the modified second Zagreb connection index is

$$
Z C_{2}^{*}(H)=1740 n+1124 .
$$

$$
\begin{aligned}
Z C_{2}^{*}(G)= & \sum_{p q \in E(G)}\left[d G(p) \tau_{G}(q)+d_{G}(q) \tau_{G}(p)\right] \\
= & \sum_{p q \in E_{2,3}^{c}}\left[d_{H}(p) \tau_{H}(q)+d_{H}(q) \tau_{H}(p)\right]+\sum_{p q \in E_{3,3}^{c}}\left[d_{H}(p) \tau_{H}(q)+d_{H}(q) \tau_{H}(p)\right] \\
& +\sum_{p q \in E_{3,5}^{c}}\left[d_{H}(p) \tau_{H}(q)+d_{H}(q) \tau_{H}(p)\right]+\sum_{p q \in E_{4,5}^{c}}\left[d_{H}(p) \tau_{H}(q)+d_{H}(q) \tau_{H}(p)\right] \\
& +\sum_{p q \in E_{4,4}^{c}}\left[d_{H}(p) \tau_{H}(q)+d_{H}(q) \tau_{H}(p)\right]+\sum_{p q \in E_{3,4}^{c}}\left[d_{H}(p) \tau_{H}(q)+d_{H}(q) \tau_{H}(p)\right] \\
& +\sum_{p q \in E_{4,4}^{c}}\left[d_{H}(p) \tau_{H}(q)+d_{H}(q) \tau_{H}(p)\right]+\sum_{p q \in E_{5,8}^{c}}\left[d_{H}(p) \tau_{H}(q)+d_{H}(q) \tau_{H}(p)\right] \\
= & \left|E_{2,3}^{C}\right|(2 \times 3+2 \times 2)+\left|E_{3,3}^{C}\right|(2 \times 3+2 \times 3)+\left|E_{3,5}^{C}\right|(2 \times 5+3 \times 3)+\left|E_{4,5}^{C}\right|(2 \times 5+3 \times 4) \\
& +\left|E_{4,4}^{C}\right|(2 \times 4+3 \times 4)+\left|E_{3,4}^{C}\right|(2 \times 4+3 \times 3)+\left|E_{4,4}^{C}\right|(3 \times 4+3 \times 4)+\left|E_{5,8}^{C}\right|(3 \times 8+4 \times 5) \\
= & (4 n+12)(10)+(12 n+4)(12)+(4 n+12)(19)+(12 n+4)(22)+(12 n+4)(20) \\
& +(24 n+8)(17)+(9 n+3)(24)+(8 n+8)(44) \\
= & 40 n+120+144 n+48+76 n+228+264 n+88+240 n+80 \\
& +408 n+136+216 n+72+352 n+352 \\
= & 1740 n+1124 .
\end{aligned}
$$

Theorem 5. Let $H \cong Z N O X(n)$ be a zinc oxide network of dimensions $n \geq 3$. Then, the modified third Zagreb connection index is

$$
Z C_{3}^{*}(H)=1808 n+1184
$$

Proof. By definition,

$$
\begin{aligned}
Z C_{3}^{*}(G)= & \sum_{p q \in E(G)}\left[d_{G}(p) \tau_{G}(p)+d_{G}(q) \tau_{G}(q)\right] \\
= & \sum_{p q \in E_{2,3}^{c}}\left[d_{H}(p) \tau_{H}(p)+d_{H}(q) \tau_{H \backslash}(q)\right]+\sum_{p q \in E_{3,3}^{c}}\left[d_{H}(p) \tau_{H}(p)+d_{H}(q) \tau_{H}(q)\right] \\
& +\sum_{p q \in E_{3,5}^{c}}\left[d_{H}(p) \tau_{H \backslash}(p)+d_{H}(q) \tau_{H}(q)\right]+\sum_{p q \in E_{4,5}^{c}}\left[d_{H}(p) \tau_{H}(p)+d_{H}(q) \tau_{H}(q)\right] \\
& +\sum_{p q \in E_{4,4}^{c}}\left[d_{H}(p) \tau_{H}(p)+d_{H}(q) \tau_{H}(q)\right]+\sum_{p q \in E_{3,4}^{c}}\left[d_{H}(p) \tau_{H}(p)+d_{H}(q) \tau_{H}(q)\right] \\
& +\sum_{p q \in E_{4,4}^{c}}\left[d_{H}(p) \tau_{H}(p)+d_{H}(q) \tau_{H}(q)\right]+\sum_{p q \in E_{5,8}^{c}}\left[d_{H}(p) \tau_{H}(p)+d_{H}(q) \tau_{H}(q)\right] \\
= & \left|E_{2,3}^{c}\right|(2 \times 2+2 \times 3)+\left|E_{3,3}^{c}\right|(2 \times 3+2 \times 3)+\left|E_{3,5}^{c}\right|(2 \times 3+3 \times 5)+\left|E_{4,5}^{c}\right|(2 \times 4+3 \times 5) \\
& +\left|E_{4,4}^{c}\right|(2 \times 4+3 \times 4)+\left|E_{3,4}^{c}\right|(2 \times 3+3 \times 4)+\left|E_{4,4}^{c *}\right|(3 \times 4+3 \times 4)+\left|E_{5,8}^{c}\right|(3 \times 5+4 \times 8) \\
= & (4 n+12)(10)+(12 n+4)(12)+(4 n+12)(21)+(12 n+4)(23)+(12 n+4)(20) \\
& +(24 n+8)(18)+(9 n+3)(24)+(8 n+8)(47) \\
= & 40 n+120+144 n+48+84 n+252+276 n+92+240 n+80 \\
& +432 n+144+216 n+72+376 n+376 \\
= & 1808 n+1184 .
\end{aligned}
$$


Theorem 6. Let $H \cong Z N O X(n)$ be a zinc oxide network of dimensions $n \geq 3$. Then, the modified fourth Zagreb connection index is

$$
Z C_{4}^{*}(H)=10344 n+7224
$$

$$
\begin{aligned}
Z C_{4}^{*}(G)= & \sum_{p q \in E(G)}\left[d_{G}(p) \tau_{G}(q) \times d_{G}(q) \tau_{G}(p)\right] \\
= & \sum_{p q \in E_{2,3}^{c}}\left[d_{H}(p) \tau_{H}(q) \times d_{H}(q) \tau_{H}(p)\right]+\sum_{p q \in E_{3,3}^{c}}\left[d_{H}(p) \tau_{H}(q) \times d_{H}(q) \tau_{H}(p)\right] \\
& +\sum_{p q \in E_{3,5}^{c}}\left[d_{H}(p) \tau_{H}(q) \times d_{H}(q) \tau_{H}(p)\right]+\sum_{p q \in E_{4,5}^{c}}\left[d_{H}(p) \tau_{H}(q) \times d_{H}(q) \tau_{H}(p)\right] \\
& +\sum_{p q \in E_{4,4}^{c}}\left[d_{H}(p) \tau_{H}(q) \times d_{H}(q) \tau_{H}(p)\right]+\sum_{p q \in E_{3,4}^{c}}\left[d_{H}(p) \tau_{H}(q) \times d_{H}(q) \tau_{H}(p)\right] \\
& +\sum_{p q \in E_{4,4}^{c}}\left[d_{H}(p) \tau_{H}(q) \times d_{H}(q) \tau_{H}(p)\right]+\sum_{p q \in E_{5,8}^{c}}\left[d_{H}(p) \tau_{H}(q) \times d_{H}(q) \tau_{H}(p)\right] \\
= & \left|E_{2,3}^{c}\right|(2 \times 3 \times 2 \times 2)+\left|E_{3,3}^{c}\right|(2 \times 3 \times 2 \times 3)+\left|E_{3,5}^{c}\right|(2 \times 5 \times 3 \times 3)+\left|E_{4,5}^{c}\right|(2 \times 5 \times 3 \times 4) \\
& +\left|E_{4,4}^{c}\right|(2 \times 4 \times 3 \times 4)+\left|E_{3,4}^{c}\right|(2 \times 4 \times 3 \times 3)+\left|E_{4,4}^{C *}\right|(3 \times 4 \times 3 \times 4)+\left|E_{5,8}^{C}\right|(3 \times 8 \times 4 \times 5) \\
= & (4 n+12)(24)+(12 n+4)(36)+(4 n+12)(90)+(12 n+4)(120)+(12 n+4)(96) \\
& +(24 n+8)(72)+(9 n+3)(144)+(8 n+8)(480) \\
= & 96 n+288+432 n+144+360 n+1080+1440 n+480+1152 n+384 \\
& +1728 n+576+1296 n+432+3840 n+3840 \\
= & 10344 n+7224 .
\end{aligned}
$$

\section{Main Results Based on Zinc Silicate Network $($ ZNSL $(n))$}

In this section, we compute the main results for first Zagreb connection index (ZCI), second ZCI, modified first ZCI, modified second ZCI, modified third ZCI, and modified fourth ZCI of zinc silicate-related $\operatorname{MON}(\mathrm{ZNSL}(n))$. Let $K \cong$ ZNSL $(n)$ be the zinc silicate network of dimension $n$ in the plane, see Figure 2. The partitions of $K$ with respect to the vertex set and edge set are $V(K)$ and $E(K)$. We can easily see each vertex of degrees 2, 3, and 4. We have $V_{1}=\left\{v \in V(K) \mid d_{v}\right.$ $=2\}, \quad V_{2}=\left\{v \in V(K) \mid d_{v}=3\right\}$, and $V_{3}=\left\{v \in V(K) \mid d_{v}=4\right\}$, where $\left|V_{1}\right|=42 n+30,\left|V_{2}\right|=38 n+18$, and $\left|V_{3}\right|=2 n+2$. So, $\mid$ $V(K)|=v=| V_{1}|+| V_{2}|+| V_{3} \mid=82 n+50$. Now, the partitions of vertices according to connection number are $V_{1}=\{v \in V$ $\left.(K) \mid \tau_{v}=2\right\}, \quad V_{2}=\left\{v \in V(K) \mid \tau_{v}=3\right\}, \quad V_{3}=\left\{v \in V(K) \mid \tau_{v}=4\right\}$, $V_{4}=\left\{v \in V \quad(K) \mid \tau_{v}=5\right\}, \quad V_{5}=\left\{v \in V \quad(K) \mid \tau_{v}=6\right\}, \quad$ and $V_{6}=\left\{v \in V(K) \mid \tau_{v}=8\right\}$, where $\left|V_{1}\right|=2 n+6,\left|V_{2}\right|=16 n+16$, $\mid$
$V_{3}|=48 n+16,| V_{4}|=8 n+8,| V_{5} \mid=6 n+2$, and $\left|V_{6}\right|=2 n+2$. So, $\quad|V \quad(K)|=v=\left|V_{1}\right|+\left|V_{2}\right|+\left|V_{3}\right|+\left|V_{4}\right|+\left|V_{5}\right|=82 n+50$. These vertex partitions are shown in Tables 5 and 6 .

There are four types of partitions of edge sets of $K$ according to the degree as $\mid E$ $(K)|=| E_{2,2}^{d}|+| E_{2,3}^{d}|+| E_{3,3}^{d}|+| E_{3,4}^{d} \mid=103 n+61$, and there are seven types of partitions of edge sets of Kaccording to the connection number of vertices as $|E(K)|=\left|E_{2,3}^{c}\right|+$ $\left|E_{3,3}^{c}\right|+\left|E_{3,4}^{c}\right|+\left|E_{3,5}^{c}\right|+\left|E_{4,4}^{c}\right|+\left|E_{4,5}^{c}\right|+\left|E_{4,6}^{c}\right|+\left|E_{5,8}^{c}\right|+\left|E_{6,6}^{c}\right|=$ $103 n+61$. These edge partitions are shown in Tables 7 and 8 .

Theorem 7. Let $K \cong Z N S L(n)$ be a zinc silicate network of dimensions $n \geq 3$. Then, the first Zagreb connection index is

$$
Z C_{1}(K)=1464 n+824 \text {. }
$$

Proof. By definition,

$$
\begin{aligned}
Z C_{1}(G) & =\sum_{q \in V(G)}\left[\tau_{G}(q)\right]^{2} \\
& =(2 n+6)(2)^{2}+(16 n+16)(3)^{2}+(48 n+16)(4)^{2}+(8 n+8)(5)^{2}+(6 n+2)(6)^{2}+(2 n+2)(8)^{2} \\
& =8 n+24+144 n+144+768 n+256+200 n+200+216 n+72+128 n+128 \\
& =1464 n+824 .
\end{aligned}
$$


TABle 5: Partitions of $K$ 's vertices according to the degree.

\begin{tabular}{lccc}
\hline$d_{v}$ & 2 & 3 & 4 \\
\hline$\left|d_{v}\right|$ & $42 n+30$ & $38 n+18$ & $2 n+2$ \\
\hline
\end{tabular}

Table 6: Partitions of $K$ 's vertices according to the connection number.

\begin{tabular}{lcccccc}
\hline$\tau_{v}$ & 2 & 3 & 4 & 5 & 6 & 8 \\
\hline$\left|\tau_{v}\right|$ & $2 n+6$ & $16 n+16$ & $48 n+16$ & $8 n+8$ & $6 n+2$ & $2 n+2$ \\
\hline
\end{tabular}

Table 7: Partitions of $K$ 's edges according to the degree.

\begin{tabular}{lcccc}
\hline$E_{d(p), d(q)}^{d}$ & $E_{2,2}^{d}$ & $E_{2,3}^{d}$ & $E_{3,3}^{d}$ & $E_{3,4}^{d}$ \\
\hline$\left|E_{d(p), d(q)}^{d}\right|$ & $10 n+14$ & $64 n+32$ & $21 n+7$ & $8 n+8$ \\
\hline
\end{tabular}

Table 8: Partition of K's edges according to the connection number.

\begin{tabular}{lccccccccc}
\hline$E_{\tau(p), \tau(q)}^{c}$ & $E_{2,3}^{c}$ & $E_{3,3}^{c}$ & $E_{3,4}^{c}$ & $E_{3,5}^{c}$ & $E_{4,4}^{c}$ & $E_{4,5}^{c}$ & $E_{4,6}^{c}$ & $E_{5,8}^{c}$ & $E_{6,6}^{c}$ \\
\hline$\left|E_{\tau(p), \tau(q)}^{c}\right|$ & $4 n+12$ & $6 n+2$ & $12 n+4$ & $4 n+12$ & $42 n+14$ & $12 n+4$ & $12 n+4$ & $8 n+8$ & $3 n+1$ \\
\hline
\end{tabular}

Theorem 8. Let $K \cong Z N S L(n)$ be a zinc silicate network of dimensions $n \geq 3$. Then, the second Zagreb connection index is

Proof. By definition,

$$
Z C_{2}(K)=1910 n+1074
$$

$$
\begin{aligned}
Z C_{2}(G)= & \sum_{p q \in E(G)}\left[\tau_{G}(p) \times \tau G(q)\right] \\
= & \sum_{p q \in E_{2,3}^{c}}\left[\tau_{K}(p) \times \tau_{K}(q)\right]+\sum_{p q \in E_{3,3}^{c}}\left[\tau_{K}(p) \times \tau_{K}(q)\right]+\sum_{p q \in E_{3,5}^{c}}\left[\tau_{K}(p) \times \tau_{K}(q)\right] \\
& +\sum_{p q \in E_{4,5}^{c}}\left[\tau_{K}(p) \times \tau_{K}(q)\right]+\sum_{p q \in E_{4,4}^{c}}\left[\tau_{K}(p) \times \tau_{K}(q)\right]+\sum_{p q \in E_{3,4}^{c}}\left[\tau_{K}(p) \times \tau_{K}(q)\right] \\
& +\sum_{p q \in E_{4,4}^{c}}\left[\tau_{K}(p) \times \tau_{K}(q)\right]+\sum_{p q \in E_{4,6}^{c}}\left[\tau_{K}(p) \times \tau_{K}(q)\right]+\sum_{p q \in E_{6,6}^{c}}\left[\tau_{K}(p) \times \tau_{K}(q)\right] \\
& +\sum_{p q \in E_{5,8}^{c}}\left[\tau_{K}(p) \times \tau_{K}(q)\right] \\
= & \left|E_{(2,3)(K)}\right|(2 \times 3)+\left|E_{(3,3)(K)}\right|(3 \times 3)+\left|E_{(3,5)(K)}\right|(3 \times 5)+\left|E_{(4,5)(K)}\right|(4 \times 5) \\
& +\left|E_{(4,4)(K)}\right|(4 \times 4)+\left|E_{(3,4)(K)}\right|(3 \times 4)+\left|E_{(4,4)(K)}\right|(4 \times 4)+\left|E_{(4,6)(K)}\right|(4 \times 6) \\
& +\left|E_{(6,6)(K)}\right|(6 \times 6)+\left|E_{(5,8)(K)}\right|(5 \times 8) \\
= & (4 n+12)(2 \times 3)+(6 n+2)(3 \times 3)+(4 n+12)(3 \times 5)+(12 n+4)(4 \times 5) \\
& +(36 n+12)(4 \times 4)+(12 n+4)(3 \times 4)+(6 n+2)(4 \times 4)+(12 n+4)(4 \times 6) \\
& +(3 n+1)(6 \times 6)+(8 n+8)(5 \times 8) \\
= & 24 n+72+54 n+18+60 n+180+240 n+80+576 n+192 \\
& +144 n+48+96 n+32+288 n+96+108 n+36+320 n+320 \\
= & 1910 n+1074 .
\end{aligned}
$$


Theorem 9. Let $K \cong Z N S L(n)$ be a zinc silicate network of dimensions $n \geq 3$. Then, the modified first Zagreb connection index is

$$
Z C_{1}^{*}(K)=876 n+500 .
$$

$$
\begin{aligned}
Z C_{1}^{*}(G)= & \sum_{p q \in E(G)}\left[\tau_{G}(p)+\tau_{G}(q)\right] \\
= & \sum_{p q \in E_{2,3}^{c}}\left[\tau_{k}(p)+\tau_{K}(q)\right]+\sum_{p q \in E_{3,3}^{c}}\left[\tau_{K}(p)+\tau_{K}(q)\right]+\sum_{p q \in E_{3,5}^{c}}\left[\tau_{K}(p)+\tau_{K}(q)\right] \\
& +\sum_{p q \in E_{4,5}^{c}}\left[\tau_{K}(p)+\tau_{K}(q)\right]+\sum_{p q \in E_{4,4}^{c}}\left[\tau_{K}(p)+\tau_{K}(q)\right]+\sum_{p q \in E_{3,4}^{c}}\left[\tau_{K}(p)+\tau_{K}(q)\right] \\
& +\sum_{p q \in E_{4,4}^{c}}\left[\tau_{K}(p)+\tau_{K}(q)\right]+\sum_{p q \in E_{4,6}^{c}}\left[\tau_{K}(p)+\tau_{K}(q)\right]+\sum_{p q \in E_{6,6}^{c}}\left[\tau_{K}(p)+\tau_{K}(q)\right] \\
& +\sum_{p q \in E_{5,8}^{c}}\left[\tau_{K}(p)+\tau_{K}(q)\right] \\
= & \left|E_{(2,3)(K)}\right|(2+3)+\left|E_{(3,3)(K)}\right|(3+3)+\left|E_{(3,5)(K)}\right|(3+5)+\left|E_{(4,5)(K)}\right|(4+5) \\
& +\left|E_{(4,4)(K)}\right|(4+4)+\left|E_{(3,4)(K)}\right|(3+4)+\left|E_{(4,4)(K)}\right|(4+4)+\left|E_{(4,6)(K)}\right|(4+6) \\
& +\left|E_{(6,6)(K)}\right|(6+6)+\left|E_{(5,8)(K)}\right|(5+8) \\
= & (4 n+12)(2+3)+(6 n+2)(3+3)+(4 n+12)(3+5)+(12 n+4)(4+5) \\
& +(36 n+12)(4+4)+(12 n+4)(3+4)+(6 n+2)(4+4)+(12 n+4)(4+6) \\
& +(3 n+1)(6+6)+(8 n+8)(5+8) \\
= & 20 n+60+36 n+12+32 n+96+108 n+36+288 n+96+84 n+28+48 n+16 \\
& +120 n+40+36 n+12+104 n+104 \\
= & 876 n+500 .
\end{aligned}
$$

Theorem 10. Let $K \cong Z N S L(n)$ be a zinc silicate network of

$$
Z C_{2}^{*}(K)=2340 n+1324
$$
dimensions $n \geq 3$. Then, the modified second Zagreb connection index is

Proof. By definition, 


$$
\begin{aligned}
Z C_{2}^{*}(G)= & \sum_{p q \in E(G)}\left[d_{G}(p) \tau_{G}(q)+d_{G}(q) \tau_{G}(p)\right] \\
= & \sum_{p q \in E_{2,3}^{c}}\left[d_{K}(p) \tau_{K}(q)+d_{K}(q) \tau_{K}(p)\right]+\sum_{p q \in E_{3,3}^{c}}\left[d_{K}(p) \tau_{K}(q)+d_{K}(q) \tau_{K}(p)\right] \\
& +\sum_{p q \in E_{3,5}^{c}}\left[d_{K}(p) \tau_{K}(q)+d_{K}(q) \tau_{K}(p)\right]+\sum_{p q \in E_{4,5}^{c}}\left[d_{K}(p) \tau_{K}(q)+d_{K}(q) \tau_{K}(p)\right] \\
& +\sum_{p q \in E_{4,4}^{c}}\left[d_{K}(p) \tau_{K}(q)+d_{K}(q) \tau_{K}(p)\right]+\sum_{p q \in E_{3,4}^{c}}\left[d_{K}(p) \tau_{K}(q)+d_{K}(q) \tau_{K}(p)\right] \\
& +\sum_{p q \in E_{4,4}^{c *}}\left[d_{K}(p) \tau_{K}(q)+d_{K}(q) \tau_{K}(p)\right]+\sum_{p q \in E_{4,6}^{c}}\left[d_{K}(p) \tau_{K}(q)+d_{K}(q) \tau_{K}(p)\right] \\
& +\sum_{p q \in E_{6,6}^{c}}\left[d_{K}(p) \tau_{K}(q)+d_{K}(q) \tau_{K}(p)\right]+\sum_{p q \in E_{5,8}^{c}}\left[d_{K}(p) \tau_{K}(q)+d_{K}(q) \tau_{K}(p)\right] \\
= & E_{2,3}^{c}|(2 \times 3+2 \times 2)+| E_{3,3}^{c}|(2 \times 3+2 \times 3)+| E_{3,5}^{c}|(2 \times 5+3 \times 3)+| E_{4,5}^{c}|(2 \times 5+3 \times 4)+| E_{4,4}^{c} \mid(2 \times 4+3 \times 4) \\
& +\left|E_{3,4}^{c}\right|(2 \times 4+3 \times 3)+\left|E_{4,4}^{c *}\right|(3 \times 4+3 \times 4)+\left|E_{4,6}^{c}\right|(3 \times 6+3 \times 4)+\left|E_{6,6}^{c}\right|(3 \times 6+3 \times 6)+\left|E_{5,8}^{c}\right|(3 \times 8+4 \times 5) \\
= & (4 n+12)(10)+(6 n+2)(12)+(4 n+12)(19)+(12 n+4)(22)+(36 n+12)(20)+(12 n+4)(17) \\
& +(6 n+2)(24)+(12 n+4)(30)+(3 n+1)(36)+(8 n+8)(44) \\
= & 40 n+120+72 n+24+76 n+228+264 n+88+720 n+240+204 n+68+144 n+48+360 n+120 \\
& +108 n+36+352 n+352 \\
= & 2340 n+1324 .
\end{aligned}
$$

Theorem 11. Let $K \cong Z N S L(n)$ be a zinc silicate network of dimensions $n \geq 3$. Then, the modified third Zagreb connection index is

$$
Z C_{3}^{*}(K)=2396 n+1380
$$

Proof. By definition,

$$
\begin{aligned}
Z C_{3}^{*}(G)= & \sum_{p q \in E(G)}\left[d_{G}(p) \tau_{G}(p)+d_{G}(q) \tau_{G}(q)\right] \\
= & \sum_{p q \in E_{2,3}^{c}}\left[d_{K}(p) \tau_{K}(p)+d_{K}(q) \tau_{K}(q)\right]+\sum_{p q \in E_{3,3}^{c}}\left[d_{K}(p) \tau_{K}(p)+d_{K}(q) \tau_{K}(q)\right] \\
& +\sum_{p q \in E_{3,5}^{c}}\left[d_{K}(p) \tau_{K}(p)+d_{K}(q) \tau_{K}(q)\right]+\sum_{p q \in E_{4,5}^{c}}\left[d_{K}(p) \tau_{K}(p)+d_{K}(q) \tau_{K}(q)\right] \\
& +\sum_{p q \in E_{4,4}^{c}}\left[d_{K}(p) \tau_{K}(p)+d_{K}(q) \tau_{K}(q)\right]+\sum_{p q \in E_{3,4}^{c}}\left[d_{K}(p) \tau_{K}(p)+d_{K}(q) \tau_{K}(q)\right] \\
& +\sum_{p q \in E_{4,4}^{c *}}\left[d_{K}(p) \tau_{K}(p)+d_{K}(q) \tau_{K}(q)\right]+\sum_{p q \in E_{4,6}^{c}}\left[d_{K}(p) \tau_{K}(p)+d_{K}(q) \tau_{K}(q)\right] \\
& +\sum_{p q \in E_{6,6}^{c}}\left[d_{K}(p) \tau_{K}(p)+d_{K}(q) \tau_{K}(q)\right]+\sum_{p q \in E_{5,8}^{c}}\left[d_{K}(p) \tau_{K}(p)+d_{K}(q) \tau_{K}(q)\right] \\
= & E_{2,3}^{c}|(2 \times 2+2 \times 3)+| E_{3,3}^{c}|(2 \times 3+2 \times 3)+| E_{3,5}^{c}|(2 \times 3+3 \times 5)+| E_{4,5}^{c}|(2 \times 4+3 \times 5)+| E_{4,4}^{c} \mid(2 \times 4+3 \times 4) \\
& +\left|E_{3,4}^{c}\right|(2 \times 3+3 \times 4)+\left|E_{4,4}^{c *}\right|(3 \times 4+3 \times 4)+\left|E_{4,6}^{c}\right|(3 \times 4+3 \times 6)+\left|E_{6,6}^{c}\right|(3 \times 6+3 \times 6)+\left|E_{5,8}^{c}\right|(3 \times 5+4 \times 8) \\
= & (4 n+12)(10)+(6 n+2)(12)+(4 n+12)(21)+(12 n+4)(23)+(36 n+12)(20)+(12 n+4)(18) \\
& +(6 n+2)(24)+(12 n+4)(30)+(3 n+1)(36)+(8 n+8)(47) \\
= & 40 n+120+72 n+24+84 n+252+276 n+92+720 n+240+216 n+72+144 n+48+360 n \\
& +120+108 n+36+376 n+376 \\
= & 2396 n+1380 .
\end{aligned}
$$


Theorem 12. Let $K \cong Z N S L(n)$ be a zinc silicate network of dimensions $n \geq 3$. Then, the modified fourth Zagreb connection index is

$$
Z C_{4}^{*}(K)=14700 n+8676
$$

$$
\begin{aligned}
& Z C_{4}^{*}(G)=\sum_{p q \in E(G)}\left[d_{G}(p) \tau_{G}(q) \times d_{G}(q) \tau_{G}(p)\right] \\
& =\sum_{p q \in E_{2,3}^{c}}\left[d_{K}(p) \tau_{K}(q) \times d_{K}(q) \tau_{K}(p)\right]+\sum_{p q \in E_{3,3}^{c}}\left[d_{K}(p) \tau_{K}(q) \times d_{K}(q) \tau_{K}(p)\right] \\
& +\sum_{p q \in E_{3,5}^{c}}\left[d_{K}(p) \tau_{K}(q) \times d_{K}(q) \tau_{K}(p)\right]+\sum_{p q \in E_{4,5}^{c}}\left[d_{K}(p) \tau_{K}(q) \times d_{K}(q) \tau_{K}(p)\right] \\
& +\sum_{p q \in E_{4,4}^{c}}\left[d_{K}(p) \tau_{K}(q) \times d_{K}(q) \tau_{K}(p)\right]+\sum_{p q \in E_{3,4}^{c}}\left[d_{K}(p) \tau_{K}(q) \times d_{K}(q) \tau_{K}(p)\right] \\
& +\sum_{p q \in E_{4,4}^{c *}}\left[d_{K}(p) \tau_{K}(q) \times d_{K}(q) \tau_{K}(p)\right]+\sum_{p q \in E_{4,6}^{c}}\left[d_{K}(p) \tau_{K}(q) \times d_{K}(q) \tau_{K}(p)\right] \\
& +\sum_{p q \in E_{6,6}^{c}}\left[d_{K}(p) \tau_{K}(q) \times d_{K}(q) \tau_{K}(p)\right]+\sum_{p q \in E_{5,8}^{c}}\left[d_{K}(p) \tau_{K}(q) \times d_{K}(q) \tau_{K}(p)\right] \\
& =\left|E_{2,3}^{c}\right|(2 \times 3 \times 2 \times 2)+\left|E_{3,3}^{c}\right|(2 \times 3 \times 2 \times 3)+\left|E_{3,5}^{c}\right|(2 \times 5 \times 3 \times 3)+\left|E_{4,5}^{c}\right|(2 \times 5 \times 3 \times 4)+\left|E_{4,4}^{c}\right|(2 \times 4 \times 3 \times 4) \\
& +\left|E_{3,4}^{c}\right|(2 \times 4 \times 3 \times 3)+\left|E_{4,4}^{c *}\right|(3 \times 4 \times 3 \times 4)+\left|E_{4,6}^{c}\right|(3 \times 6 \times 3 \times 4)+\left|E_{6,6}^{c}\right|(3 \times 6 \times 3 \times 6)+\left|E_{5,8}^{c}\right|(3 \times 8 \times 4 \times 5) \\
& =(4 n+12)(24)+(6 n+2)(36)+(4 n+12)(90)+(12 n+4)(120)+(36 n+12)(96)+(12 n+4)(72) \\
& +(6 n+2)(144)+(12 n+4)(216)+(3 n+1)(324)+(8 n+8)(480) \\
& =96 n+288+216 n+72+360 n+1080+1440 n+480+3456 n+1152 \\
& +864 n+288+864 n+288+2592 n+864+972 n+324+3840 n+3840 \\
& =14700 n+8676 \text {. }
\end{aligned}
$$

\section{Comparisons and Conclusions}

In this section, we compare zinc oxide $(\mathrm{H})$ and zinc silicate (K) related $\mathrm{MONs}$ via some Zagreb connection indices (ZCIs) such as first ZCI, second ZCI, modified first ZCI, modified second ZCI, modified third ZCI, and modified fourth ZCI with the help of Tables 9-14 that have been constructed by using numerical values of the aforementioned ZCIs. The graphical presentations for ZCIs of MONs are presented in Figures 3-10.

The comparative study of zinc-related MONs is highlighted by the following conclusions:

(i) From Tables 9-14 and Figures 3-8, we see that the behaviors for all the ZCIs of zinc silicate MONs have more values and upper lines than zinc oxide MONs with the following order:

$Z C_{1}(K) \geq Z C_{1}(H), Z C_{2}(K) \geq Z C_{2}(H), Z C_{1}^{*}(K) \geq$ $Z C_{1}^{*}(H), Z C_{2}^{*}(K) \geq Z C_{2}^{*}(H), Z C_{3}^{*}(K) \geq Z C_{3}^{*}(H)$, and $Z C_{4}^{*}(K) \geq Z C_{4}^{*}(H)$.

(ii) From Tables 15 and 16 and Figures 9 and 10, we see that modified fourth $\mathrm{ZCI}\left(Z C_{4}^{*}\right)$ attains more values and upper lines than other ZCIs for both zinc-related MONs.

(iii) The modified first Zagreb connection index $\left(Z C_{1}^{*}\right)$ attained better values of correlation coefficient for the thirteen physicochemical properties of octane isomers than other classical Zagreb indices. In this paper, novel connection-based Zagreb index $Z C_{4}^{*}$ attains better values of correlation coefficient for increasing order in both the cases of zinc-related MONs.

(iv) Table 17 shows that zinc silicate-related MON of dimension $n$ for the aforesaid ZCIs has attained upward position than zinc oxide-related MON.

(v) Moreover, these general relations (Tables 15-17) indicate that the chemical capability of zinc silicaterelated MON is better than zinc oxide-related MON for all values of $n$.

Now, the problem is still open for prism, product, subdivision, and their compliment networks with the help of connection-based Zagreb indices. 
TAвLe 9: Numerical values of $Z C_{1}$ for $H$ and $K$ networks on dimensions $3 \leq n \leq 10$.

\begin{tabular}{lcccccccc}
\hline$Z C I s$ & 3 & 4 & 5 & 6 & 7 & 8 & 9 & 10 \\
\hline$Z C_{1}(H)$ & 3896 & 4964 & 6032 & 7100 & 8168 & 9236 & 10304 & 11372 \\
$Z C_{1}(K)$ & 5216 & 6680 & 8144 & 9608 & 11072 & 12536 & 14000 & 15464 \\
\hline
\end{tabular}

TABle 10: Numerical values of $Z C_{2}$ for $H$ and $K$ networks on dimensions $3 \leq n \leq 10$.

\begin{tabular}{lcccccccc}
\hline ZCIs & 3 & 4 & 5 & 6 & 7 & 8 & 9 & 10 \\
\hline$Z C_{2}(H)$ & 5024 & 6400 & 7776 & 9152 & 10528 & 11904 & 13280 & 14656 \\
$Z C_{2}(K)$ & 6804 & 8714 & 10624 & 12534 & 14444 & 16354 & 18264 & 20174 \\
\hline
\end{tabular}

TABLE 11: Numerical values of $Z C_{1}^{*}$ for $H$ and $K$ networks on dimensions $3 \leq n \leq 10$.

\begin{tabular}{lcccccccc}
\hline ZCIs & 3 & 4 & 5 & 6 & 7 & 8 & 9 & 10 \\
\hline$Z C_{1}^{*}(H)$ & 2448 & 3120 & 3792 & 4464 & 5136 & 5808 & 6480 & 7152 \\
$Z C_{1}^{*}(K)$ & 3128 & 4004 & 4880 & 5756 & 6632 & 7508 & 8384 & 9260 \\
\hline
\end{tabular}

TABLE 12: Numerical values of $Z C_{2}^{*}$ for $H$ and $K$ networks on dimensions $3 \leq n \leq 10$.

\begin{tabular}{lcccccccc}
\hline ZCIs & 3 & 4 & 5 & 6 & 7 & 8 & 9 & 10 \\
\hline$Z C_{2}^{*}(H)$ & 6344 & 8084 & 9824 & 11564 & 13304 & 15044 & 16784 & 18524 \\
$Z C_{2}^{*}(K)$ & 8344 & 10684 & 13024 & 15364 & 17704 & 20044 & 22384 & 24724 \\
\hline
\end{tabular}

TABLe 13: Numerical values of $Z C_{3}^{*}$ for $H$ and $K$ networks on dimensions $3 \leq n \leq 10$.

\begin{tabular}{lcccccccc}
\hline ZCIs & 3 & 4 & 5 & 6 & 7 & 8 & 9 & 10 \\
\hline$Z C_{3}^{*}(H)$ & 6608 & 8416 & 10224 & 12032 & 13840 & 15648 & 17456 & 19264 \\
$Z C_{3}^{*}(K)$ & 8568 & 10964 & 13360 & 15756 & 18152 & 20548 & 22944 & 25340 \\
\hline
\end{tabular}

TABLE 14: Numerical values of $Z C_{4}^{*}$ for $H$ and $K$ networks on dimensions $3 \leq n \leq 10$.

\begin{tabular}{lcccccccc}
\hline ZCIs & 3 & 4 & 5 & 6 & 7 & 8 & 9 & 10 \\
\hline$Z C_{4}^{*}(H)$ & 38256 & 48600 & 58944 & 69288 & 79632 & 89976 & 100320 & 110664 \\
$Z C_{4}^{*}(K)$ & 52776 & 67476 & 82176 & 96876 & 111576 & 126276 & 140976 & 155676 \\
\hline
\end{tabular}

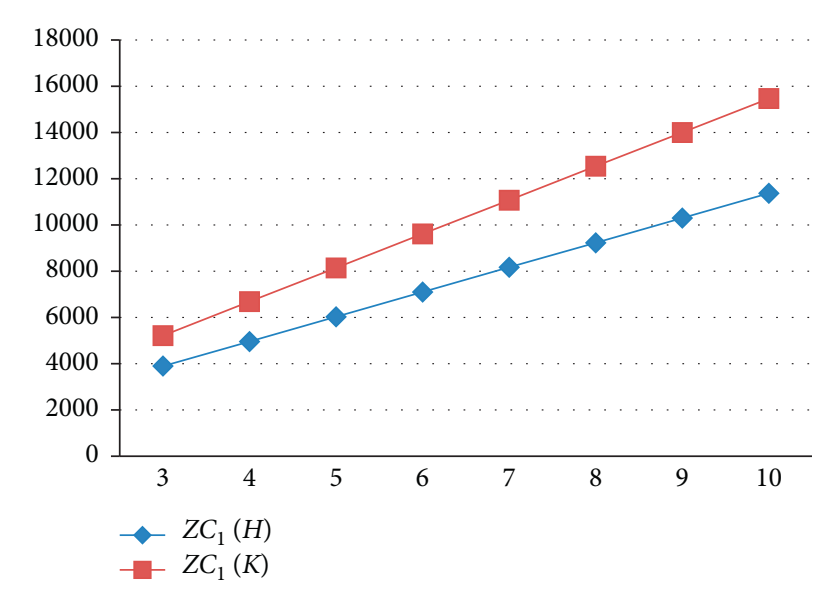

FIgure 3: Comparison of $Z C_{1}$ index for $H$ and $K$ networks on dimensions $3 \leq n \leq 10$. 


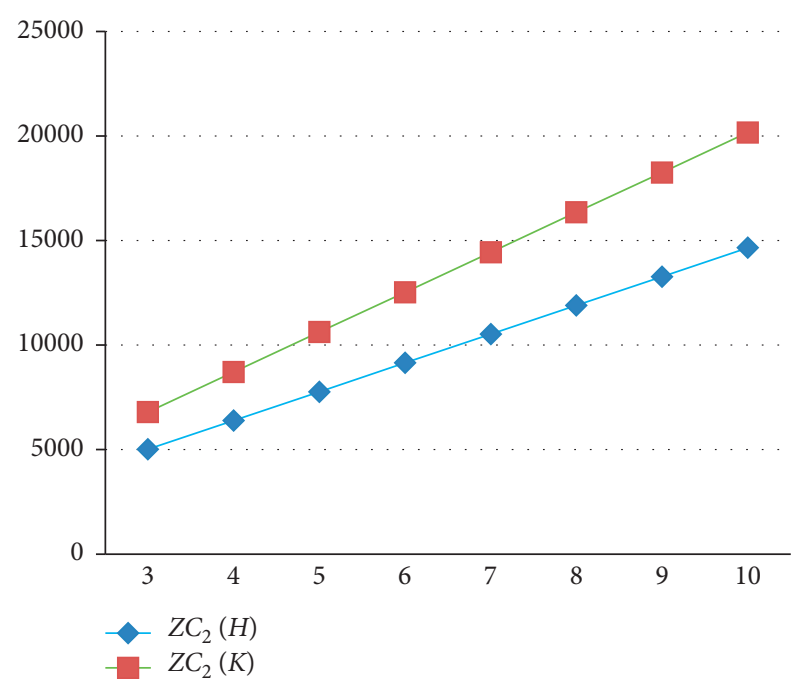

Figure 4: Comparison of $Z C_{2}$ index for $H$ and $K$ networks on dimensions $3 \leq n \leq 10$.

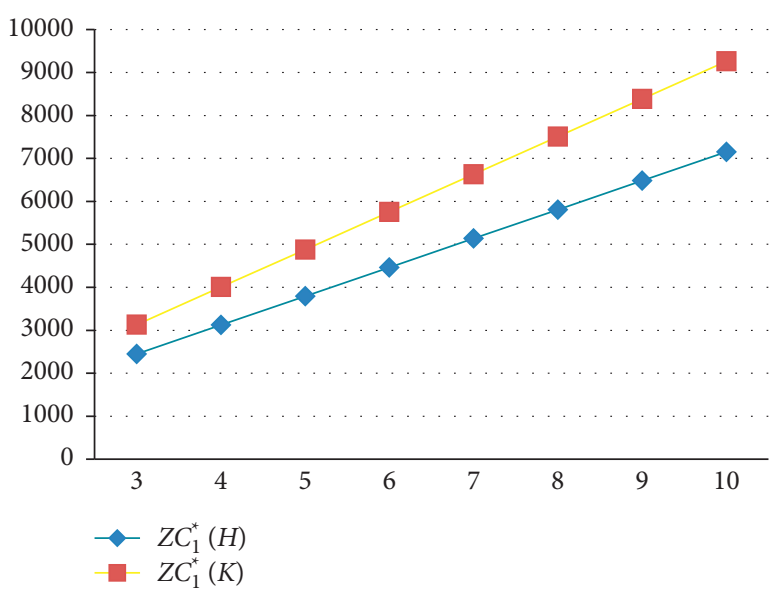

Figure 5: Comparison of $Z C_{1}^{*}$ index for $H$ and $K$ networks on dimensions $3 \leq n \leq 10$.

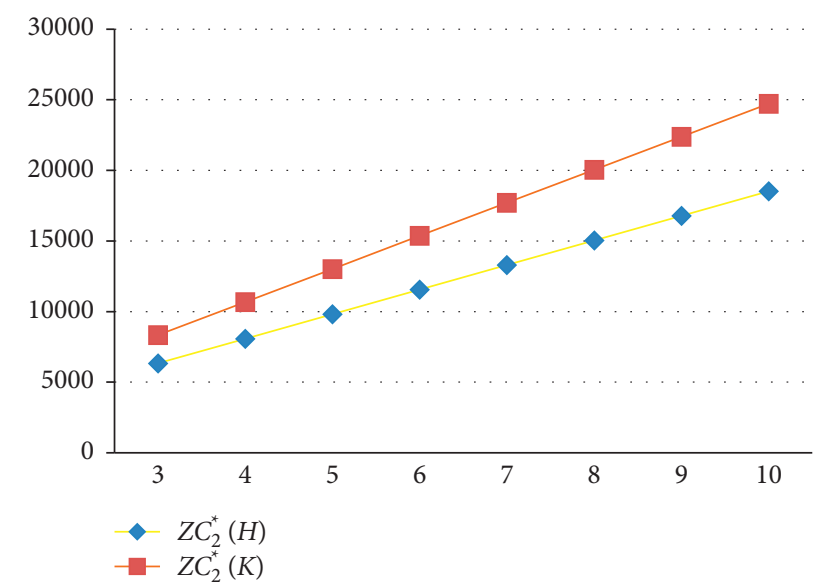

Figure 6: Comparison of $Z C_{2}^{*}$ index for $H$ and $K$ networks on dimensions $3 \leq n \leq 10$.

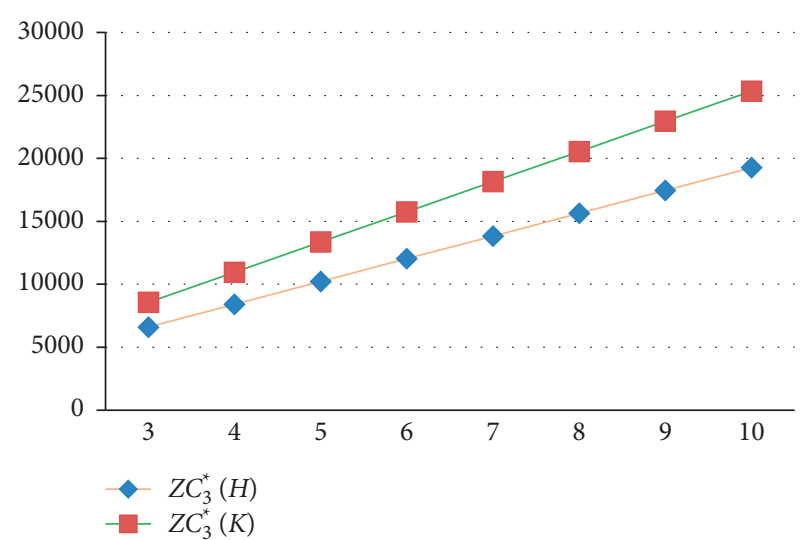

Figure 7: Comparison of $Z C_{3}^{*}$ index for $H$ and $K$ networks on dimensions $3 \leq n \leq 10$

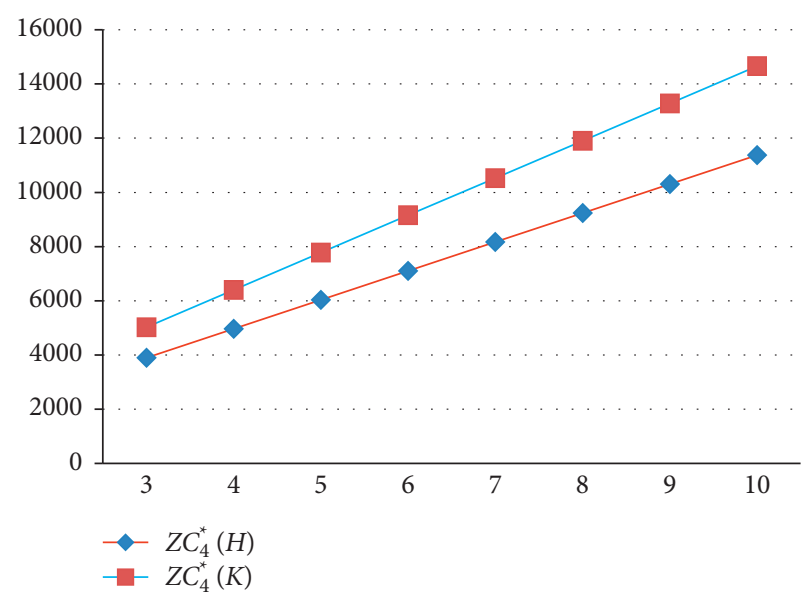

Figure 8: Comparison of $Z C_{4}^{*}$ index for $H$ and $K$ networks on dimensions $3 \leq n \leq 10$.

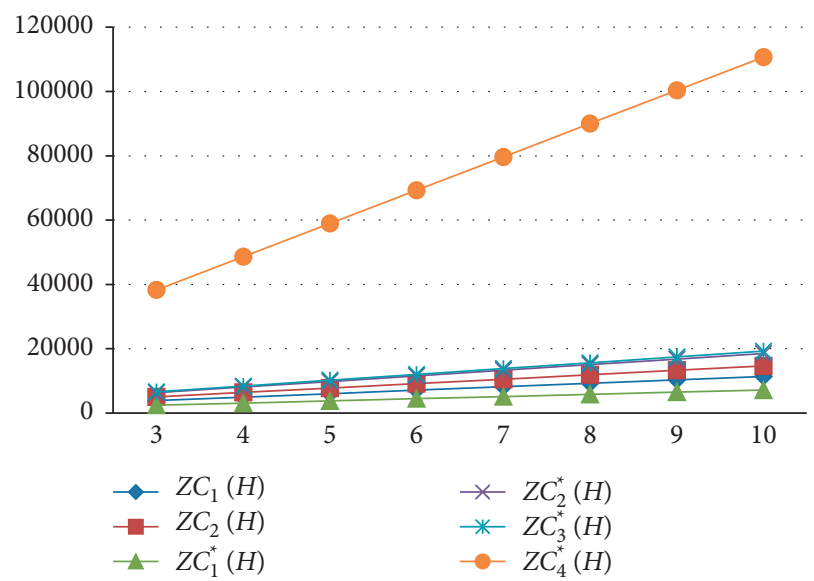

FIgure 9: Comparison of ZCIs of network $H$ on dimensions $3 \leq n \leq 10$. 


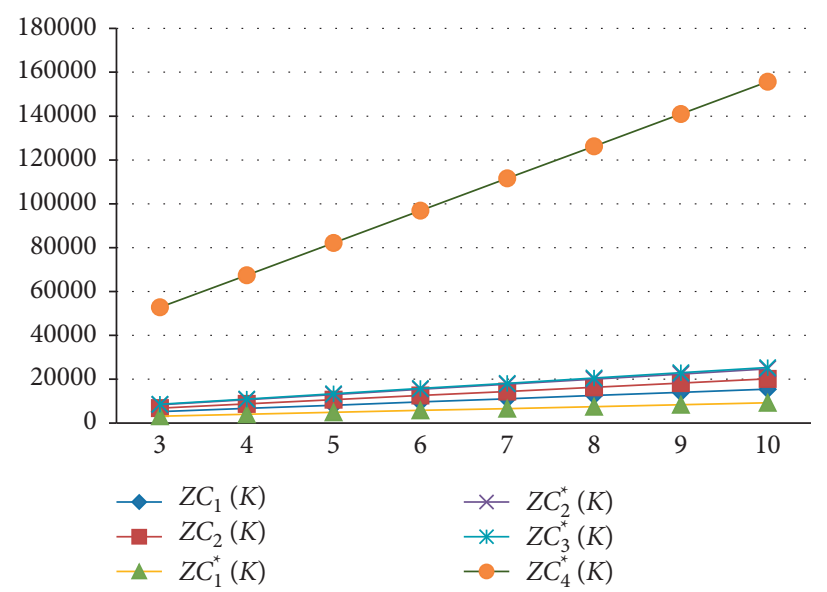

Figure 10: Comparison of ZCIs of network $K$ on dimensions $3 \leq n \leq 10$.

TABLe 15: Numerical table for indicated ZCIs of network $H$ on dimensions $3 \leq n \leq 10$.

\begin{tabular}{|c|c|c|c|c|c|c|}
\hline ZCIs & $Z C_{1}(H)$ & $Z C_{2}(H)$ & $Z C_{1}^{*}(H)$ & $Z C_{2}^{*}(H)$ & $Z C_{3}^{*}(H)$ & $Z C_{4}^{*}(H)$ \\
\hline 3 & 3896 & 5024 & 2448 & 6344 & 6608 & 38256 \\
\hline 4 & 4964 & 6400 & 3120 & 8084 & 8416 & 48600 \\
\hline 5 & 6032 & 7776 & 3792 & 9824 & 10224 & 58944 \\
\hline 6 & 7100 & 9152 & 4464 & 11564 & 12032 & 69288 \\
\hline 7 & 8168 & 10528 & 5136 & 13304 & 13840 & 79632 \\
\hline 8 & 9236 & 11904 & 5808 & 15044 & 15648 & 89976 \\
\hline 9 & 10304 & 13280 & 6480 & 16784 & 17456 & 100320 \\
\hline 10 & 11372 & 14656 & 7152 & 18524 & 19264 & 110664 \\
\hline
\end{tabular}

TABLe 16: Numerical table for indicated ZCIs of network $K$ on dimensions $3 \leq n \leq 10$.

\begin{tabular}{|c|c|c|c|c|c|c|}
\hline ZCIs & $Z C_{1}(K)$ & $Z C_{2}(K)$ & $Z C_{1}^{*}(K)$ & $Z C_{2}^{*}(K)$ & $Z C_{3}^{*}(K)$ & $Z C_{4}^{*}(K)$ \\
\hline 3 & 5216 & 6804 & 3128 & 8344 & 8568 & 52776 \\
\hline 4 & 6680 & 8714 & 4004 & 10684 & 10964 & 67476 \\
\hline 5 & 8144 & 10624 & 4880 & 13024 & 13360 & 82176 \\
\hline 6 & 9608 & 12534 & 5756 & 15364 & 15756 & 96876 \\
\hline 7 & 11072 & 14444 & 6632 & 17704 & 18152 & 111576 \\
\hline 8 & 12536 & 16354 & 7508 & 20044 & 20548 & 126276 \\
\hline 9 & 14000 & 18264 & 8384 & 22384 & 22944 & 140976 \\
\hline 10 & 15464 & 20174 & 9260 & 24724 & 25340 & 155676 \\
\hline
\end{tabular}

TABLE 17: Comparison of indicated ZCIs for all $n$.

\begin{tabular}{lcc}
\hline ZCIs & $K-H=$ ZNSL $(n)-$ ZNOX $(n)$ & Results \\
\hline First ZCI & $396 n+132$ & $K>H$ \\
Second ZCI & $534 n+178$ & $K>H$ \\
Modified first ZCI & $204 n+68$ & $K>H$ \\
Modified second ZCI & $600 n+200$ & $K>H$ \\
Modified third ZCI & $588 n+196$ & $K>H$ \\
Modified fourth ZCI & $4356 n+1452$ & $K>H$ \\
\hline
\end{tabular}




\section{Data Availability}

All data are included within this article. However, the reader may contact the corresponding author for more details of the data.

\section{Conflicts of Interest}

The authors declare that they have no conflicts of interest.

\section{References}

[1] T. B. Celic, M. Mazaj, N. Guillou, V. Kaucic, and N. Z. Logar, "New zinc-based metal organic framework material," in Proceedings of the 3rd Croatian-Solovenian Symposium on Zeolites, Trogir, Croatia, September 2010.

[2] P. Horcajada, R. Gref, T. Baati et al., "Metal-organic frameworks in biomedicine," Chemical Reviews, vol. 112, no. 2, pp. 1232-1268, 2012.

[3] Y. Cui, H. Xu, Y. Yue et al., "A luminescent mixed-lanthanide metal-organic framework thermometer," Journal of the American Chemical Society, vol. 134, no. 9, pp. 3979-3982, 2012.

[4] C. Wang, L. Tian, W. Zhu et al., "Dye@bio-MOF-1 composite as a dual-emitting platform for enhanced detection of a wide range of explosive molecules," ACS Applied Materials \& Interfaces, vol. 9, no. 23, pp. 20076-20085, 2017.

[5] H. Lu, J. Sun, H. Zhang, S. Lu, and W. C. H. Choy, "Roomtemperature solution-processed and metal oxide-free nanocomposite for the flexible transparent bottom electrode of perovskite solar cells," Nanoscale, vol. 8, no. 11, pp. 5946-5953, 2016.

[6] A. S. Prasad, "Zinc in human health: effect of zinc on immune cells," Molecular Medicine, vol. 14, no. 5-6, pp. 353-357, 2008.

[7] S. Bahrani, S. A. Hashemi, S. M. Mousavi, and R. Azhdari, "Zinc-based metal-organic frameworks as nontoxic and biodegradable platforms for biomedical applications: review study," Drug Metabolism Reviews, vol. 51, no. 3, pp. 356-377, 2019.

[8] M. Eddaoudi, J. Kim, N. Rosi et al., "Systematic design of pore size and functionality in isoreticular MOFs and their application in methane storage," Science, vol. 295, no. 5554, pp. 469-472, 2002.

[9] J. Feng, Z. Yang, D. Yang et al., "E-beam evaporated $\mathrm{Nb}_{2} \mathrm{O}_{5}$ as an effective electron transport layer for large flexible perovskite solar cells," Nanomaterials and Energy, vol. 36, pp. 1-8, 2017.

[10] S. Keskin and D. S. Sholl, "Efficient methods for screening of metal organic framework membranes for gas separations using atomically detailed models," Langmuir, vol. 25, no. 19, pp. 11786-11795, 2009.

[11] I. Ahmad and S. H. Jhung, "Composites of metal-organic frameworks: preparation and application in adsorption," Materials Today, vol. 17, no. 3, pp. 136-146, 2014.

[12] H. Li, L. Li, R.-B. Lin et al., Porous Metal-Organic Frameworks for Gas Storage and Separation: Status and Challenges, Elsevier, Amsterdam, Netherlands, 2019.

[13] Y. K. Hwang, D.-Y. Hong, J.-S. Chang et al., "Amine grafting on coordinatively unsaturated metal centers of MOFs: consequences for catalysis and metal encapsulation," Angewandte Chemie International Edition, vol. 47, no. 22, pp. 4144-4148, 2008.

[14] A. W. Thornton, K. M. Nairn, J. M. Hill, A. J. Hill, and M. R. Hill, "Metal-Organic frameworks impregnated with magnesium-decorated fullerenes for methane and hydrogen storage," Journal of the American Chemical Society, vol. 131, no. 30, pp. 10662-10669, 2009.

[15] M. Kim, J. F. Cahill, H. Fei, K. A. Prather, and S. M. Cohen, "Postsynthetic ligand and cation exchange in robust metalorganic frameworks," Journal of the American Chemical Society, vol. 134, no. 43, pp. 18082-18088, 2012.

[16] Z. Yin, Y.-L. Zhou, M.-H. Zeng, and M. Kurmoo, "The concept of mixed organic ligands in metal-organic frameworks: design, tuning and functions," Dalton Transactions, vol. 44, no. 12, pp. 5258-5275, 2015.

[17] G. Ding, J. Yuan, F. Jin et al., "High-performance all-polymer nonfullerene solar cells by employing an efficient polymersmall molecule acceptor alloy strategy," Nanomaterials and Energy, vol. 36, pp. 356-365, 2017.

[18] M. H. Yap, K. L. Fow, and G. Z. Chen, "Synthesis and applications of MOF-derived porous nanostructures," Green Energy \& Environment, vol. 2, no. 3, pp. 218-245, 2017.

[19] R.-B. Lin, S. Xiang, H. Xing, W. Zhou, and B. Chen, "Exploration of porous metal-organic frameworks for gas separation and purification," Coordination Chemistry Reviews, vol. 378, pp. 87-103, 2019.

[20] S. Klavzar and I. Gutman, "Selected properties of the Schultz molecular topological index," Journal of Chemical Information and Computer Sciences, vol. 36, pp. 1001-1003, 1996.

[21] G. Rücker and C. Rücker, "On topological indices, boiling points, and cycloalkanes," Journal of Chemical Information and Computer Sciences, vol. 39, no. 5, pp. 788-802, 1999.

[22] H. Gonzalez-Diaz, S. Vilar, L. Santana, and E. Uriarte, "Medicinal chemistry and bioinformatics - current trends in drugs discovery with networks topological indices," Current Topics in Medicinal Chemistry, vol. 7, no. 10, pp. 1015-1029, 2007.

[23] H. Weiner, "Prediction of isomeric difference in paraffin properties," Journal of the American Chemical Society, vol. 69, pp. 17-20, 1947.

[24] I. Gutman and N. Trinajstić, "Graph theory and molecular orbitals. Total $\varphi$-electron energy of alternant hydrocarbons," Chemical Physics Letters, vol. 17, no. 4, pp. 535-538, 1972.

[25] D. Zhao, Y.-M. Chu, M. K. Siddiqui et al., "On reverse degree based topological indices of polycyclic metal organic network," Polycyclic Aromatic Compounds, pp. 1-18, 2021.

[26] J.-H. Tang, U. Ali, M. Javaid, and K. Shabbir, "Zagreb connection indices of subdivision and semi-total point operations on graphs," Journal of Chemistry, vol. 2020, Article ID 9846913, 14 pages, 2020.

[27] J. Cao, U. Ali, M. Javaid, and C. Huang, "Zagreb connection indices of molecular graphs based on operations," Complexity, vol. 2020, Article ID 7385682, 15 pages, 2020.

[28] U. Ali, M. Javaid, and A. M. Alanazi, "Computing analysis of connection-based indices and coindices for product of molecular networks," Symmetry, vol. 12, no. 8, Article ID 1320, 2020.

[29] I. Gutman, B. Ruscic, N. Trinajstic, and C. F. Wilson, "Graph theory and molecular orbitals. XII. Acyclic polyenes," The Journal of Chemical Physics, vol. 62, no. 9, pp. 3399-3405, 1975.

[30] B. Furtula and I. Gutman, "A forgotten topological index," Journal of Mathematical Chemistry, vol. 53, no. 4, pp. 1184-1190, 2015.

[31] I. Gutman and O. Polansky, Mathematical Concepts in Organic Chemistry, Springer-Verlag, Berlin, Germany, 1986.

[32] A. Ali and N. Trinajstic, "A novel/old modification of the first Zagreb index,” Mol. Inform.vol. 37, pp. 1-7, 2018. 
[33] M. Javaid, U. Ali, and J.-B. Liu, "Computing first Zagreb connection index and coindex of resultant graphs," Mathematical Problems in Engineering, vol. 2021, Article ID 6019517, 19 pages, 2021.

[34] R. Todeschini and V. Consonni, Handbook of Molecular Descriptors, Wiley VCH, Weinheim, Germany, 2002.

[35] M. Javaid, U. Ali, and K. Siddiqui, "Novel connection based Zagreb indices of several wheel-related graphs," Comp. J. Combin. Math.vol. 1, pp. 1-28, 2021.

[36] S. Keskin and D. S. Sholl, "Screening Metal-Organic framework materials for membrane-based methane/carbon dioxide separations," Journal of Physical Chemistry C, vol. 111, no. 38, pp. 14055-14059, 2007.

[37] S. Yamaguchi, "Estimating the Zagreb indices and the spectral radius of triangle-and-quadrangle-free connected graphs," Chemical Physics Letters, vol. 458, no. 4, pp. 396-398, 2008. 